Revue des patrimoines

4 | 2004

Les réseaux de la villégiature

\title{
Le fonds d'atelier de Marguerite Huré au Musée des Années 30 de Boulogne-Billancourt
}

Véronique David et Carole Bouvet (épouse de Mersseman)

\section{OpenEdition}

1 Journals

Édition électronique

URL : http://journals.openedition.org/insitu/2289

DOI : 10.4000/insitu.2289

ISSN : 1630-7305

Éditeur

Ministère de la Culture

Référence électronique

Véronique David et Carole Bouvet (épouse de Mersseman), « Le fonds d'atelier de Marguerite Huré au Musée des Années 30 de Boulogne-Billancourt », In Situ [En ligne], 4 | 2004, mis en ligne le 23 juin 2014, consulté le 07 septembre 2020. URL : http://journals.openedition.org/insitu/2289 ; DOI : https:// doi.org/10.4000/insitu.2289

Ce document a été généré automatiquement le 7 septembre 2020

\section{c)}

In Situ Revues des patrimoines est mis à disposition selon les termes de la licence Creative Commons Attribution - Pas d'Utilisation Commerciale - Pas de Modification 4.0 International. 


\section{Le fonds d'atelier de Marguerite Huré au Musée des Années 30 de Boulogne-Billancourt}

Véronique David et Carole Bouvet (épouse de Mersseman)

\section{Introduction}

1 La réception au Musée des Années 30 de Boulogne-Billancourt du fonds d'atelier de Marguerite Huré (1895-1967) ${ }^{1}$ constitue une reconnaissance de son œuvre qui mettra un terme, souhaitons-le, à sa mise à l'écart tant déplorée par le Père Couturier dans son «Bilan de l'époque 1920-1940" paru dans L'Art Sacré en 1948². Marguerite Huré fait partie des peintres verriers qui ont joué un rôle essentiel dans l'ouverture du vitrail à la modernité dès l'entre-deux guerres. A l'origine d'innovations formelles et techniques, elle est l'auteur, dès 1931, du premier ensemble de verrières relevant d'une esthétique abstraite à la chapelle du séminaire de Voreppe (Isère) ${ }^{3}$. Elle est recherchée pour ses talents en matière de création par les plus grands architectes de son époque - Auguste Perret dont elle fut l'interlocutrice privilégiée mais aussi Paul Tournon, Pierre Pouradier-Duteil ou Maurice Novarina - et, parallèlement, pour ses capacités de traductrice de cartons, par des artistes de renom tels Maurice Denis, George Desvallières, le Père Couturier, Valentine Reyre, Jean Bazaine ${ }^{4}$ ou par d'autres, moins connus, comme l'abbé Maurice Morel, grand défenseur de Rouault et Picasso, qui lui a fourni les cartons des vitraux du Ham (Manche) en 1944. 
Figure 1

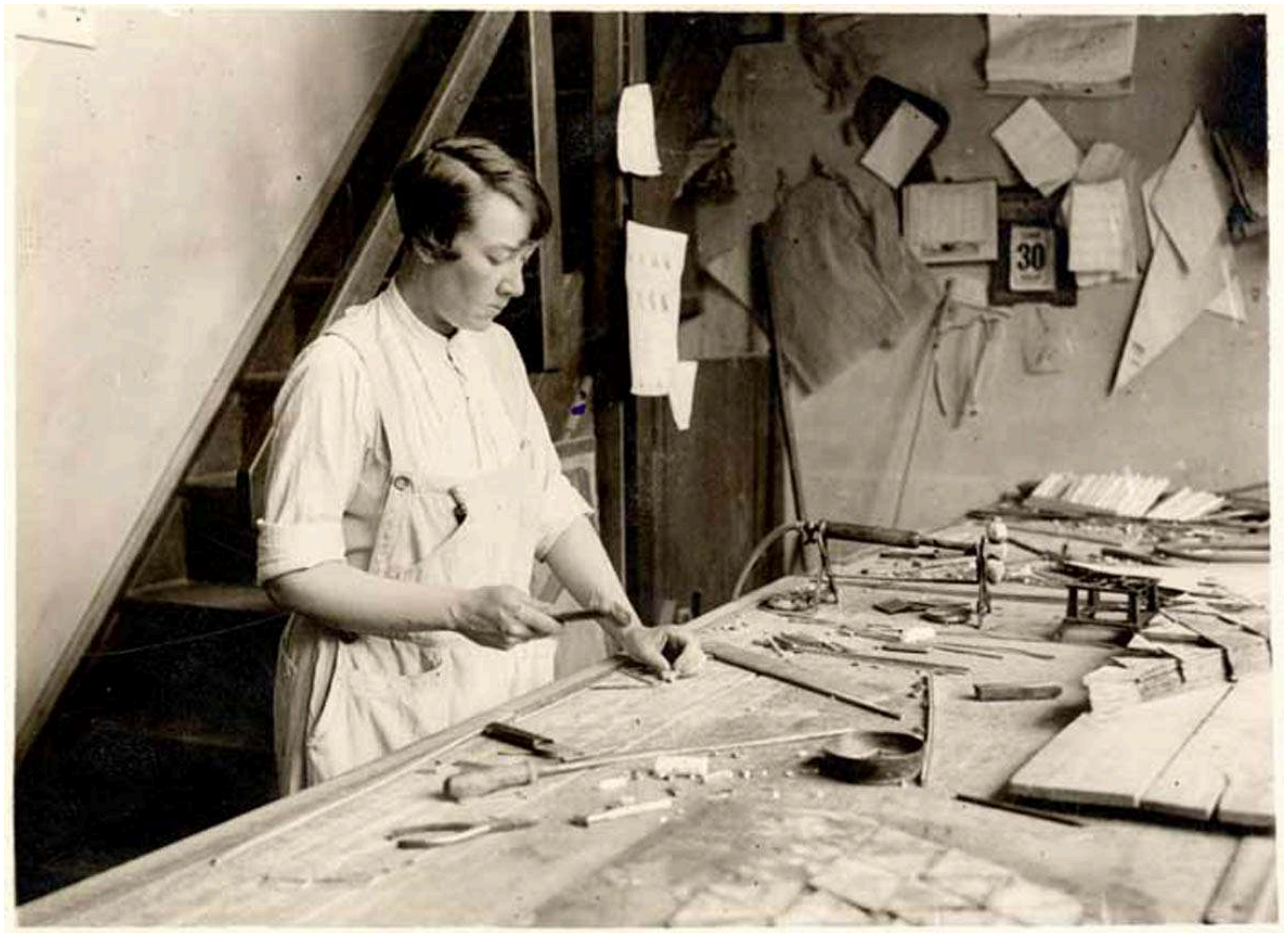

Marguerite Huré dans son atelier

Fonds Huré - Musée des Années 30 de Boulogne-Billancourt (c) Musée des Années 30 de BoulogneBillancourt

\section{Un fonds d'atelier sauvé de l'oubli}

2 La préservation du fonds d'atelier de Marguerite Huré s'inscrit dans une démarche habituelle à tout chercheur de l'Inventaire, soucieux de dépasser la connaissance des œuvres in situ pour mieux saisir la genèse de la création. La "redécouverte " ${ }^{5}$ d'une œuvre majeure de Marguerite Huré, l'ensemble de Voreppe, est à l'origine de ce questionnement: des proches de Marguerite Huré pouvaient-ils encore apporter leur témoignage et quelles étaient les sources documentaires susceptibles d'éclairer son œuvre ? (fig. $\mathbf{n}^{\circ}$ 2). La pauvreté de ces dernières fut heureusement compensée par la rencontre de Marcelle Lecamp ${ }^{6}$, collaboratrice de Marguerite Huré à partir de 1945, seule héritière de son fonds d'atelier. Inventorié par nos soins dès 1997, la question de son devenir s'est vite imposée de façon cruciale. Pour Marcelle Lecamp, il était entendu qu'il faisait partie intégrante de sa donation à une institution religieuse marseillaise comprenant comme pièce maîtresse son atelier du 93, rue de Vaugirard ${ }^{7}$. C'était pour le fonds, sinon une mort assurée, au mieux une consultation interdite à la communauté scientifique. 
Figure 2

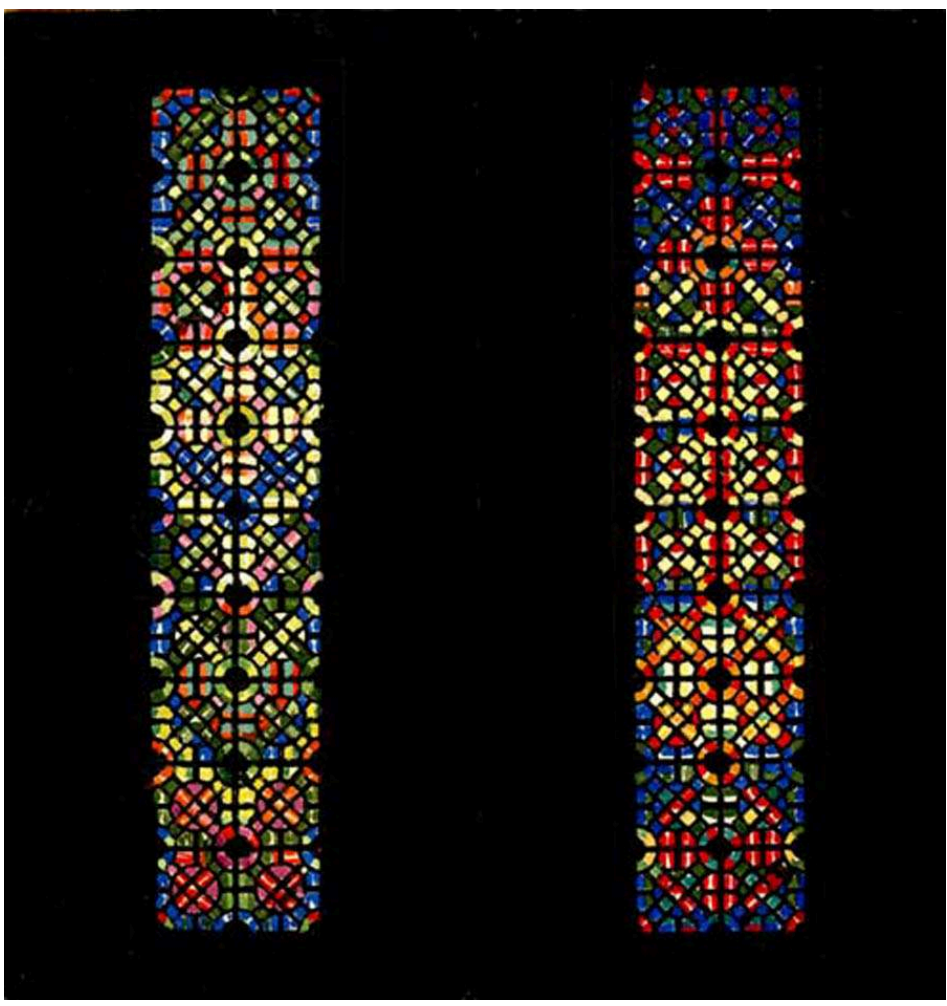

Maquettes de deux verrières : Chapelle du séminaire (actuellement lycée) de Voreppe. Inventaire Fonds Huré - Musée des Années 30 de Boulogne-Billancourt (C) Musée des Années 30 de BoulogneBillancourt. N Inv. 2003-1-28 (3 et 7) 
Figure 3

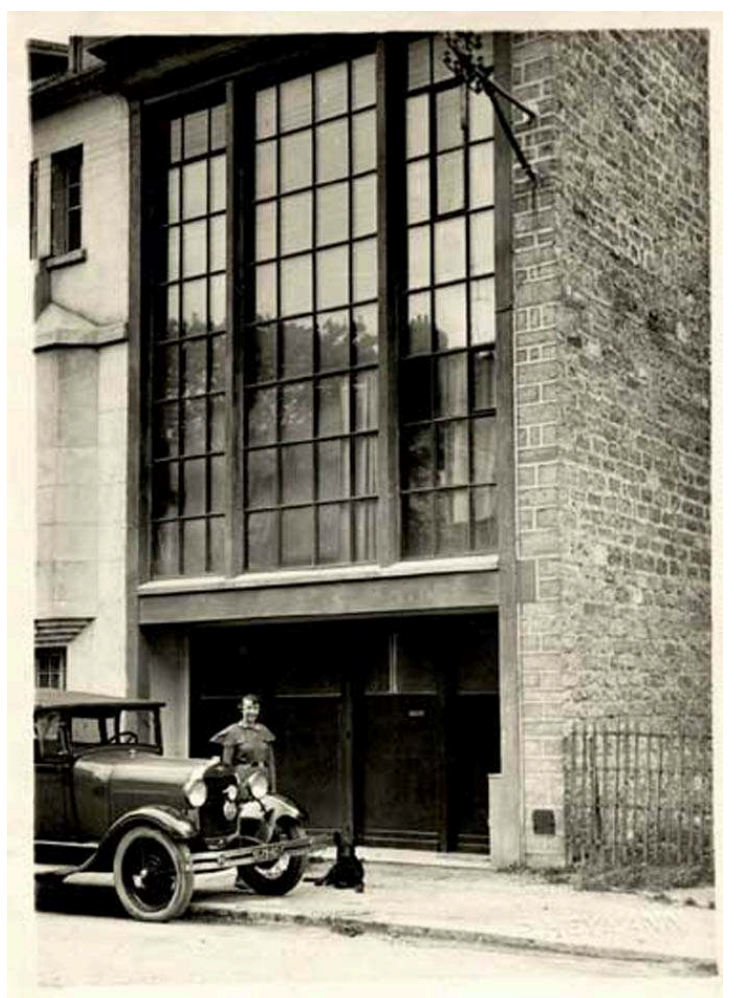

Marguerite Huré devant son atelier, 25, rue du Belvédère à Boulogne-Billancourt

Phot. P. Heymann. Fonds Huré - Musée des Années 30 de Boulogne-Billancourt @ Musée des Années 30 de Boulogne-Billancourt

3 Après examen de plusieurs solutions, comme celle d'un dépôt aux Archives du monde du travail à Roubaix ${ }^{8}$, Marcelle Lecamp accepta, sur notre suggestion, de le transmettre au Musée des Années 30 de Boulogne-Billancourt ${ }^{9}$. Ce choix se justifiait d'autant plus que Marguerite Huré y avait travaillé de 1929 à 1939, 25, rue du Belvédère, dans l'atelier que lui avait construit Auguste Perret (fig. $\mathbf{n}^{\circ} \mathbf{3}$ ). Elle y réalisa quelques-unes de ses œuvres majeures des années 30 (le Pavillon des Missions catholiques à l'Exposition coloniale internationale de 1931, les chapelles de Chalon-sur-Saône et de Voreppe etc.). Cette donation avait été soumise à deux conditions : elle ne devait être transmise qu'à la mort de Marcelle Lecamp et son propre fonds ne devait pas être dissocié de celui de Marguerite Huré ${ }^{10}$. C'est ainsi qu'à la suite du décès de Marcelle Lecamp, le 6 décembre 2000, le Musée des Années 30 de Boulogne-Billancourt a pu en hériter. En collaboration avec Carole Bouvet, attachée de conservation, nous avons procédé à des travaux de conservation préventive du fonds et à son inventaire muséologique - marquage des œuvres, constitution de dossiers d'œuvres (documentation, correspondances, articles de presse etc.) - afin de l'ouvrir à la consultation.

\section{Le fonds Huré, source de découvertes}

4 Ce fonds est essentiellement constitué de plans de coloration, d'esquisses ou de maquettes de verrières ${ }^{11}$ destinées à une trentaine d'églises paroissiales ou chapelles (lycée, collège, sanatorium, séminaire) réalisées entre 1924 et 1964. Il s'agit, pour la plupart, de constructions modernes localisées en France à l'exception de l'église de 
Sofar au Liban où, à la demande de l'architecte libanais Eddé en 1952, elle avait mis en œuvre son procédé de « briques Huré » ${ }^{12}$ Figure 4

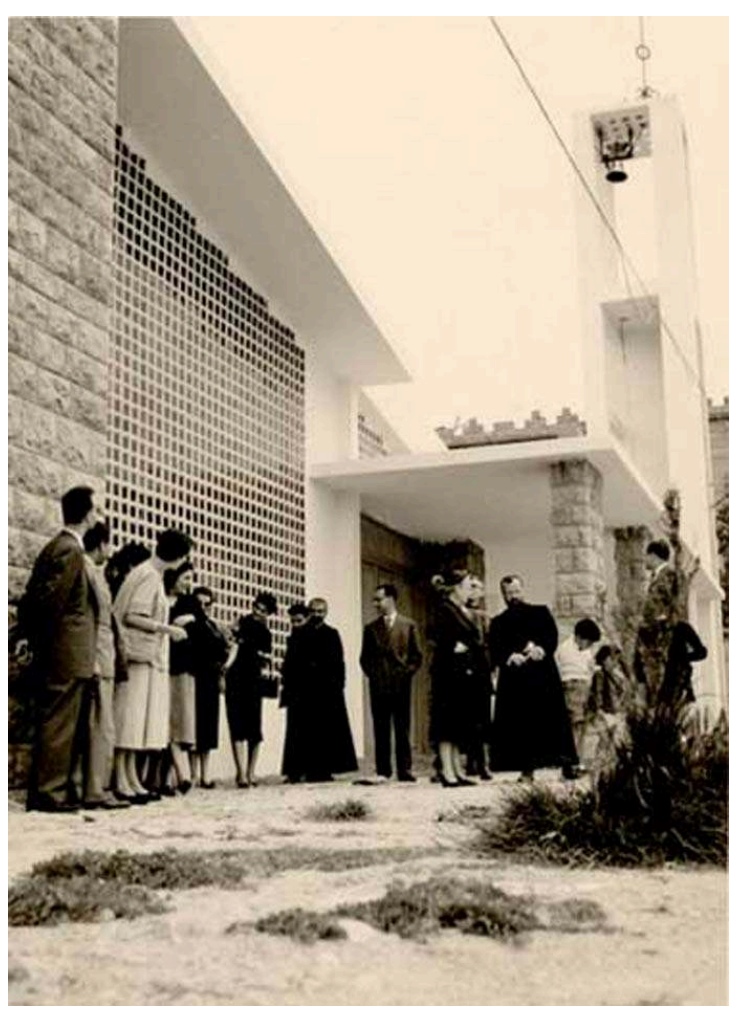

Inauguration de l'église de Sofar (Liban)

Photographie non signée. Fonds Huré - Musée des Années 30 de Boulogne-Billancourt @ Musée des Années 30 de Boulogne-Billancourt

Cet ensemble, disparu aujourd'hui, de même que la verrière des îles Chausey dans la Manche (fig. $\mathbf{n}^{\circ}$ ) ou le vitrail du Père Charles de Foucauld qui avait fasciné le Père Paul Doncoeur en 1929, lors de sa présentation à l'exposition L'art et le mobilier religieux moderne au musée Galliéra à Paris ${ }^{13}$, rend d'autant plus précieux l'existence de ce fonds. 
Figure 5

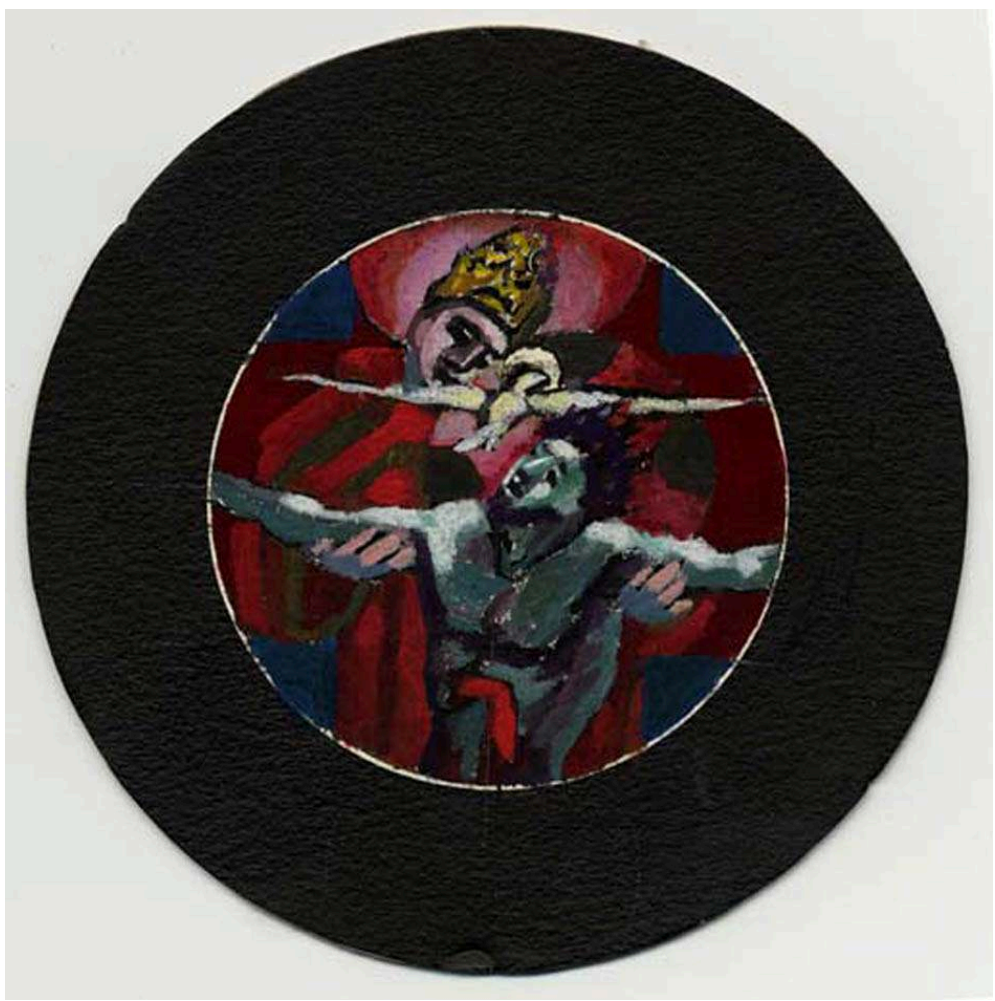

Maquette de l'oculus de « la Trinité souffrante » : Eglise îles Chausey

Fonds Huré - Musée des Années 30 de Boulogne-Billancourt @ Musée des Années 30 de Boulogne-Billancourt. $N^{\circ}$ Inv : 2003-1-11 
Figure 6

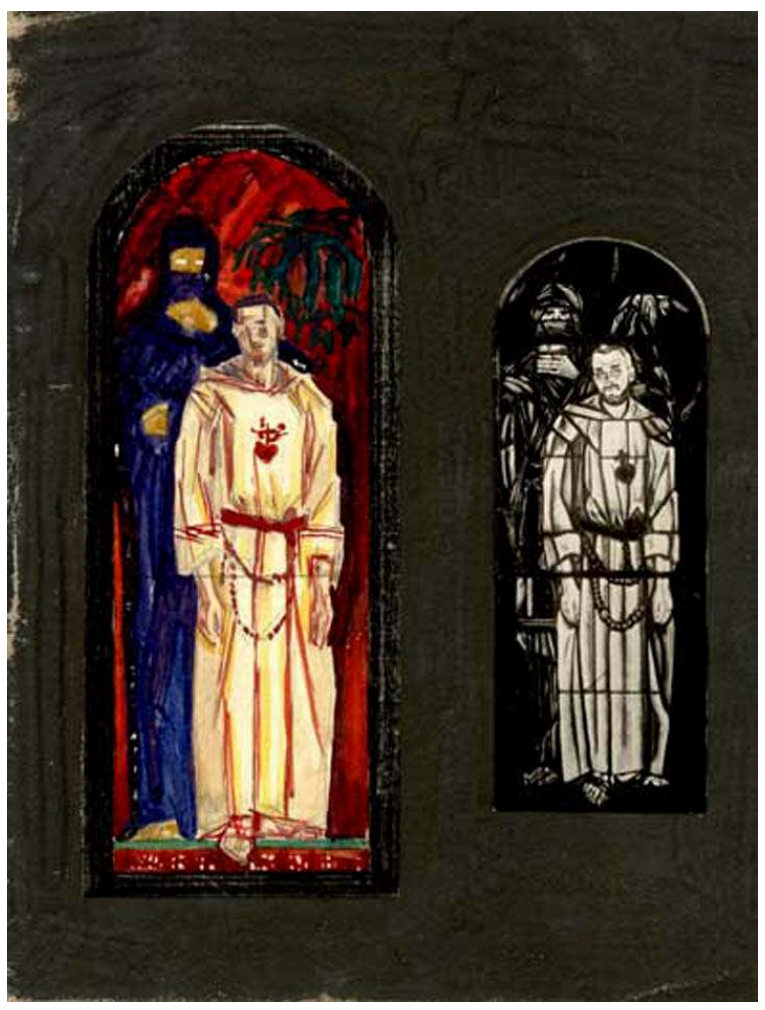

Maquette et photographie du vitrail du Père de Foucauld

Fonds Huré - Musée des Années 30 de Boulogne-Billancourt (c) Musée des Années 30 de BoulogneBillancourt. N Inv. 2003-1-26 (2)

Il révèle également l'originalité des pratiques de Marguerite Huré, tant dans le domaine technique (invention de la "brique Huré », procédé de mise au carreau des verrières) qu'esthétique. Des années 20 à 60 , elle traite ses verrières, toutes marquées par sa passion pour la couleur, aussi bien sur le mode figuratif qu'abstrait, alors même que, dans les années 50, la plupart des artistes et peintres verriers se spécialisent dans l'un ou l'autre parti.

7 Ce fonds a permis de résoudre des problèmes très importants pour la compréhension de la genèse de certaines œuvres, comme celles de l'église du Raincy (Seine-SaintDenis). Jusqu'où Marguerite Huré était-elle intervenue dans ces verrières? Personne n'ignorait que les maquettes des parties figurées avaient été réalisées par Maurice Denis, grâce à la correspondance entre Marguerite Huré et Maurice Denis conservée au Musée départemental Maurice Denis à Saint-Germain-en-Laye (Yvelines). Il était attesté qu'il l'avait encouragée à les interpréter selon sa sensibilité mais qu'en était-il des parties décoratives, exclues de ses échanges épistolaires? Deux maquettes du décor, conservées dans son fonds, apportaient déjà une réponse mais le mot dactylographié, daté du 17 janvier 1963, où Marguerite Huré relève les erreurs publiées dans le «Répertoire des nouvelles églises » publié par L’Art chrétien en 1962, se révélait encore plus explicite : « je suis nommée comme ayant exécuté les vitraux de M. Denis, lesquels, en réalité, sont des scènes tenant une petite partie des grands ensembles de couleur que j'ai conçus et exécutés " ${ }^{14}$. On peut donc la considérer comme à l'origine de ce décor qui joue un rôle capital dans l'évolution du vitrail et son ouverture à la modernité ${ }^{15}$. Son militantisme apparaît également dans les nombreux articles publiés dans les 
revues Glaces et verres, L'Architecture ou La France Catholique ainsi qu'en 1962, dans son projet, qui retiendra l'attention d'André Malraux, en faveur de la création d'un département d'enseignement et de recherches sur le vitrail à l'Ecole des Beaux-Arts de Paris.

Quant aux panneaux de vitraux qui accompagnaient le legs, les notes recueillies lors des rencontres avec Marcelle Lecamp ont permis de les identifier. On remarquera son extraordinaire "génie ailé $»^{16}$ en verre opalescent blanc ou marbré rose et vert (fig. $\mathbf{n}^{\circ}$ 7) ainsi que les vitraux pour la maison de son amie Valentine Cordier réalisés dans les années 30 .

Figure 7

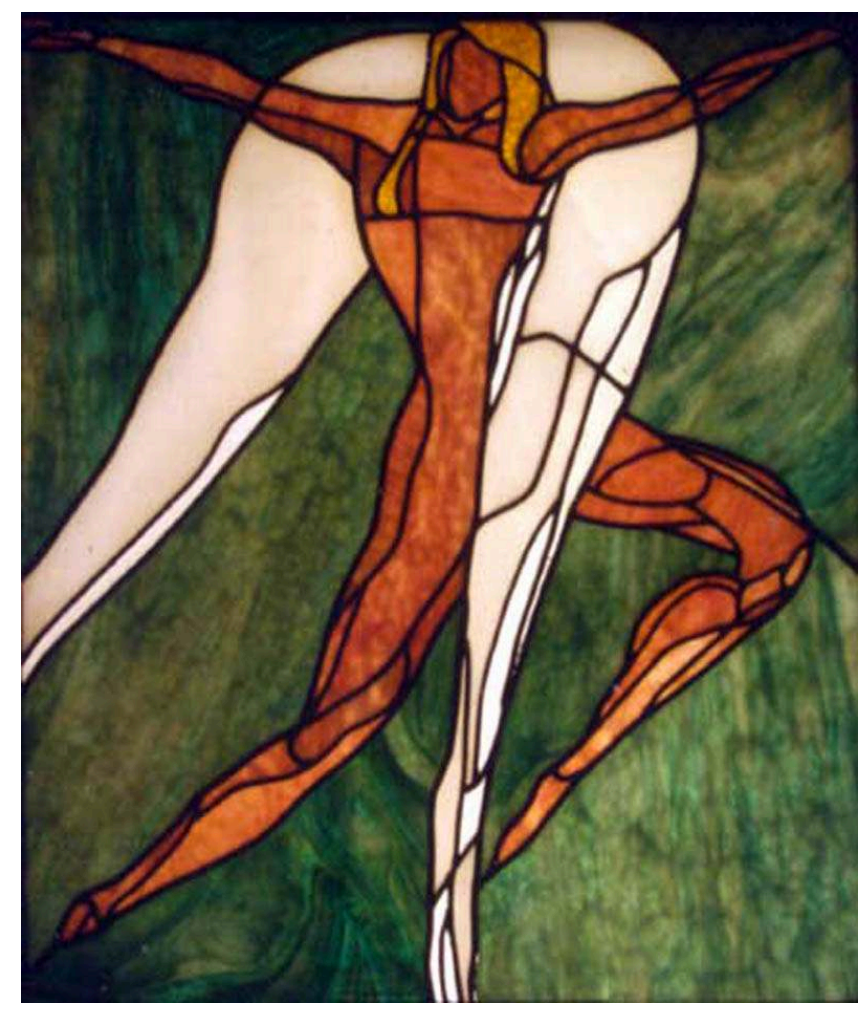

Vitrail du « Génie ailé »

Phot. Inv. P. Fortin ( Inventaire Général, ADAGP, 2003

\section{Les limites du fonds Huré}

Le fonds déposé au Musée des Années 30 ne reflète qu'une partie de l'œuvre de Marguerite Huré qui comprend une trentaine de sites supplémentaires ${ }^{17}$. Ainsi, nous n'y trouverons pas de traces de ses travaux plus modestes pour les frères Perret à la chapelle du petit séminaire Saint-Bernard de Flavigny-sur-Ozerain (Côte-d'Or) en 1930 ou bien encore à Alger, entre 1929 et $1934^{18}$. De même, ses verrières réalisées d'après les cartons d'artistes, tels Georges Desvallières pour la chapelle Saint-Yves à Paris (14 arrondissement), Wittenheim (Bas-Rhin) ou Etriché (Maine-et-Loire), Maurice Denis pour le prieuré de Saint-Germain-en-Laye (Yvelines), Fère-en-Tardenois (Aisne) ou bien encore Valentine Reyre pour Sannois (Val d'Oise) ou Audincourt (Doubs), ne sont pas représentées, les artistes ayant conservé leurs maquettes. En revanche, dans les 
dossiers d'œuvre, on peut trouver une documentation très précieuse comme cette photographie qui témoigne de la verrière commémorative de Villenauxe-la-Grande (Aube) réalisée sur un carton de Maurice Denis et détruite pendant la Seconde Guerre mondiale.

\section{L'exploitation du fonds Huré depuis son arrivée au Musée des Années 30}

10 Bien que récemment déposé au Musée des Années 30, ce fonds est déjà valorisé par son exploitation. Outre sa consultation par plusieurs chercheurs, il a été utilisé dans le cadre d'exposition et de restauration ${ }^{19}$ :

- Exposition: «Perret - la poétique du béton »: les maquettes réalisées entre 1952 et 1957 pour l'église Saint-Joseph du Havre (Seine-Maritime) ont fait partie de l'exposition «Perret, la poétique du béton » présentée en 2002 au musée Malraux du Havre puis à la GAM - Galleria Civica d'Arte Moderna e Contemporanea - de Turin en Italie. Une troisième exposition devrait avoir lieu en janvier 2004 à la Cité de l'architecture et du patrimoine au Palais de la Porte Dorée ${ }^{20}$.

- Etude préalable à la restauration des verrières du lycée François- $1^{\mathrm{er}}$ au Havre : grâce à la consultation des maquettes des verrières du lycée François- $1^{\mathrm{er}}$ au Havre, le peintre verrier Vincent Jaillette a pu réaliser l'étude préalable de restauration qui lui a été commandée en 2002 et 2003 par le Conseil régional de Haute-Normandie (fig. $\mathbf{n}^{\circ} \mathbf{8}$ ). (fig. $\mathbf{n}^{\circ}$ 9)Ces documents préparatoires permettront de remédier aux nombreuses casses provoquées par des jets de pierres tout en respectant au mieux l'aspect d'origine de cet ensemble exclusivement basé sur un jeu de couleurs et signé en 1964 par Marguerite Huré et Marcelle Lecamp. 
Figure 8

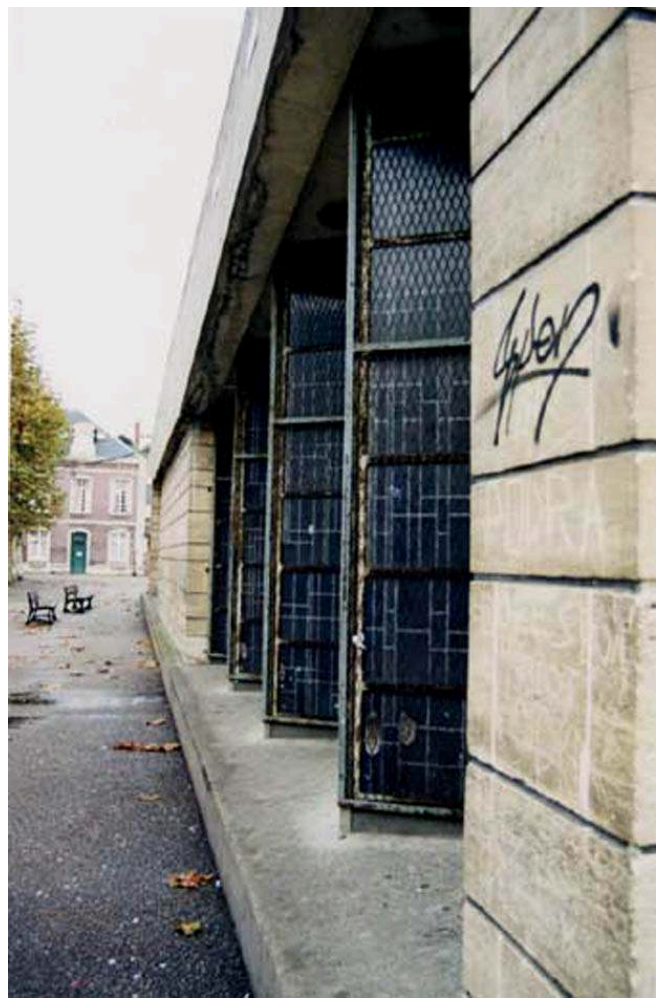

Vue extérieure des vitraux à restaurer du lycée François-7er au Havre Phot. V. Jaillette @ \. Jaillette, 2002

Figure 9

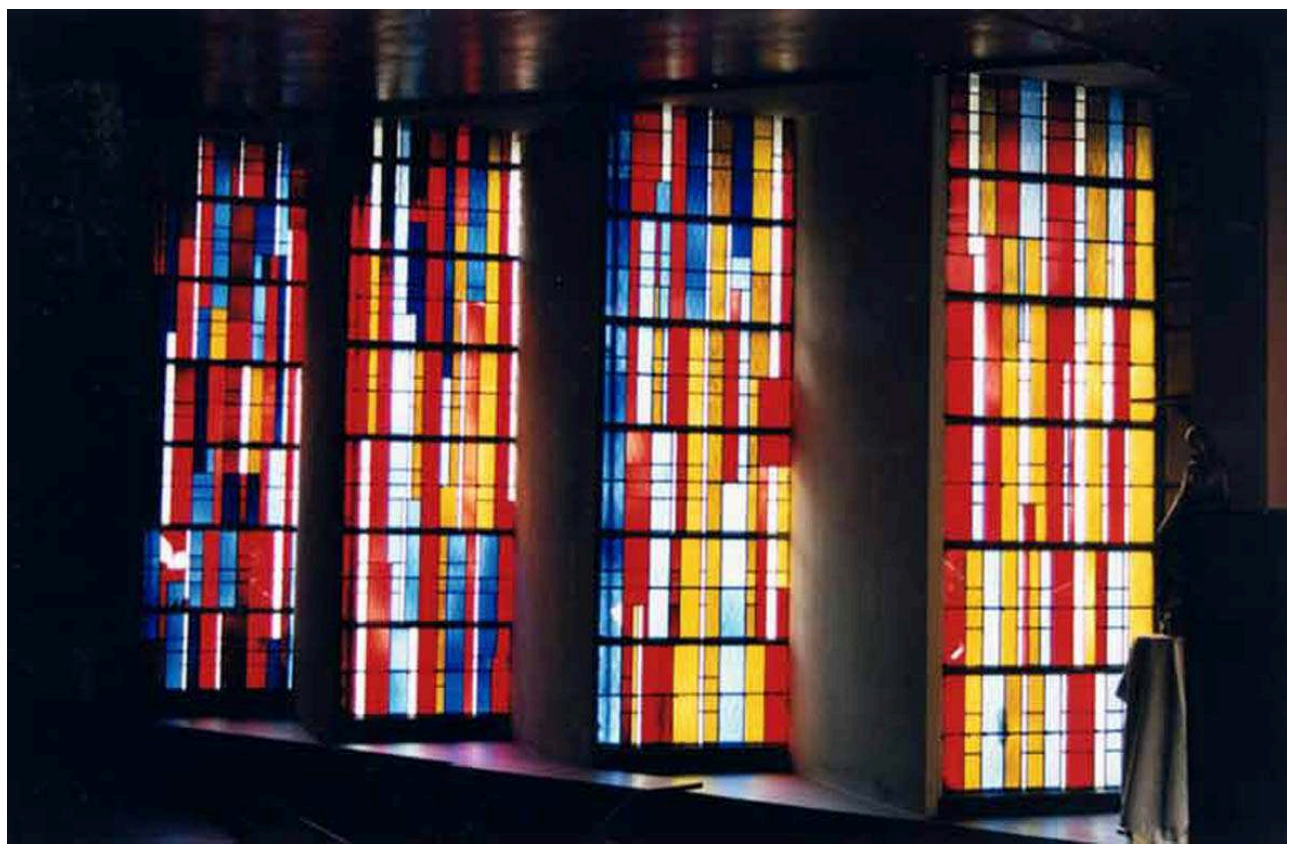

Vue intérieure des vitraux à restaurer du lycée François- † $^{\text {er }}$ au Havre Phot. V. Jaillette (c) V. Jaillette, 2002 
- Exposition d'un vitrail dans les salles du Musée des Années 30 : le vitrail de la Stigmatisation de saint François d'Assise, (fig. $\mathbf{n}^{\circ} \mathbf{1 0}$ ) peint pour la grande salle d'Afrique du Pavillon des Missions Catholiques à l'Exposition Coloniale Internationale de 1931, sera prochainement présenté dans la salle consacrée à l'art sacré du Musée des Années $30^{21}$. Conçu pour la chapelle d'Angoustrine-Villeneuve-des-Escaldes (PyrénéesOrientales), il n'a jamais été mis en place mais a figuré à l'exposition Vitraux et tapisseries modernes du Petit Palais à Paris, en $1939^{22}$, au milieu des premiers vitraux commandés par Jean Hébert-Stevens et le Père Couturier à de grands artistes d'avantgarde, Rouault, Gromaire et Bazaine. La puissance d'expression du personnage, rendue par une technique virtuose, qui n'est pas sans faire songer à la facture de Georges Desvallières fait de ce panneau l'un des chefs-d'œuvre de Marguerite Huré dans le domaine de la figuration. Sa présentation au Musée des Années 30 constitue le plus bel hommage qui pouvait lui être rendu ${ }^{23}$.

Figure 10

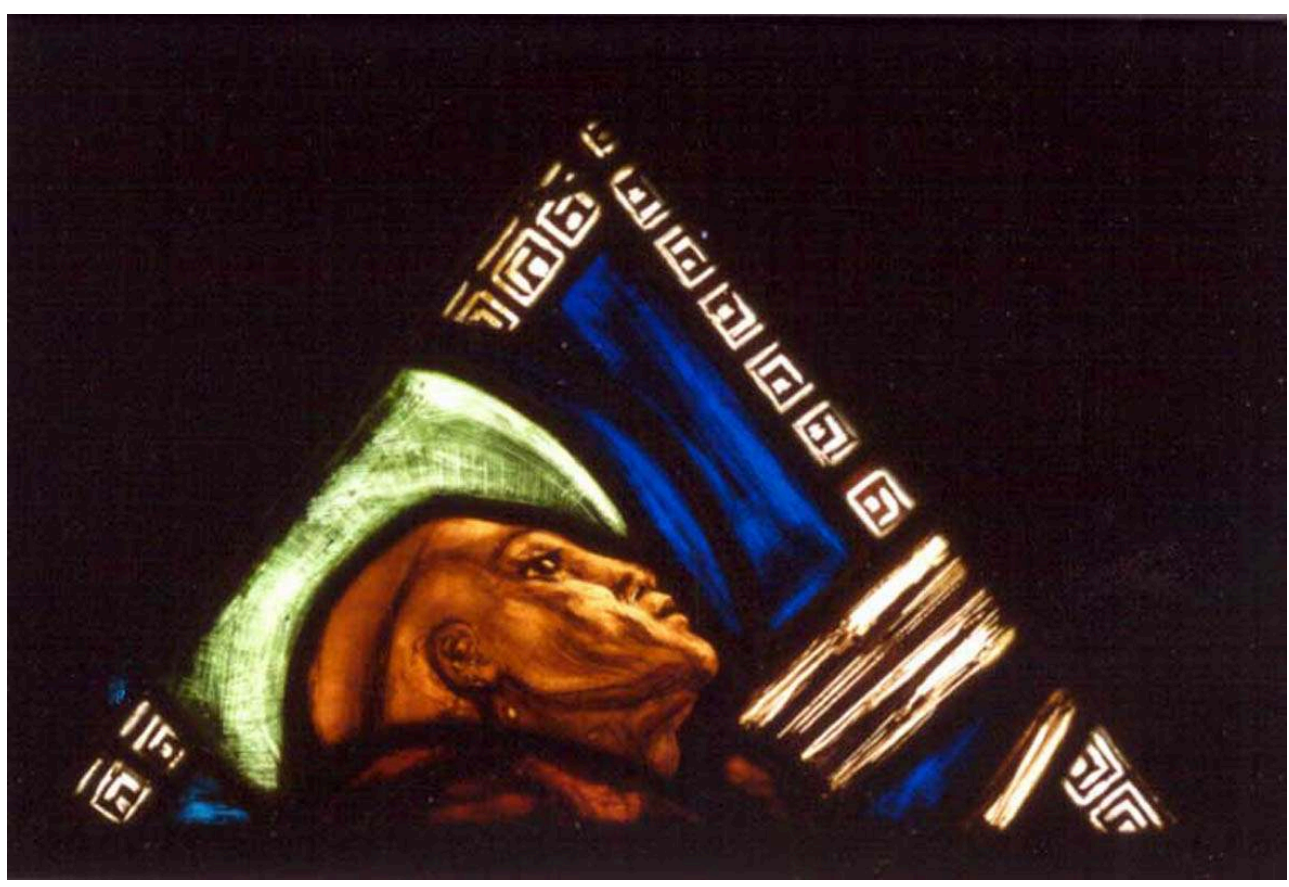

Vitrail de saint François d'Assise

Phot. Inv. P. Fortin (C) Inventaire Général, ADAGP, 2003

\section{NOTES}

1. - La vérification de son acte de naissance aux Archives de Paris a permis de rectifier l'erreur commise par le Bénézit: elle n'est pas née le 9 décembre 1896 mais le 9 décembre 1895. Elle meurt le 27 octobre 1967.

2. - L'Art Sacré. n³-4, 1948. P. 65. 
3. - David, Véronique. Marguerite Huré, précurseur de l'abstraction dans le vitrail religieux . In Situ, la revue de l'Inventaire. Paris : Ministère de la culture et de la communication, 2003, $\mathrm{n}^{\circ} 3$. 4. - David, Véronique. Marguerite Huré et les peintres. Recherches en Histoire de l'art. 2002, $\mathrm{n}^{\circ}$ 1. P. 15-27.

5. - Georges Mercier est le premier auteur à avoir mesuré l'importance des verrières de Voreppe dans l'histoire du vitrail religieux. Voir son ouvrage L'art abstrait dans l'art sacré publié à Paris en 1964 (p. 136-138 et 163).

6. - Françoise Perrot, Directeur de recherches au CNRS, nous a fait connaître l'existence de Marcelle Lecamp.

7. - Selon Marcelle Lecamp, cet atelier avait été conçu sur le même modèle que celui qu'elle avait occupé avec Marguerite Huré, entre 1939 et 1967, au 23 rue Oudinot dans le $7^{\mathrm{e}}$ arrondissement à Paris.

8. - Les Archives du monde du travail à Roubaix conservent déjà les fonds de l'atelier Le Chevallier, ainsi qu'une partie de celui de Hébert-Stevens, Peugniez et Bony.

9. - Le Musée des Années 30 de Boulogne-Billancourt a consacré une notice à l'œuvre de Marguerite Huré dans l'ouvrage L'art sacré au XX $\mathrm{XX}^{\mathrm{e}}$ siècle en France, qui a servi de catalogue à l'exposition « Le temps des Chantiers, 1920-1940 » publié en 1993 (p. 133-134).

10. - Le fonds d'atelier de Marcelle Lecamp se confond en partie avec celui de Marguerite Huré et comprend par ailleurs des maquettes de verrières réalisées par elle seule pour une vingtaine d'édifices, comme celles de Saint-Cyr-Bocage (Manche), sa première œuvre vers 1960 ou celles de Notre-Dame-du-Perpétuel-Secours à Paris (11 ${ }^{\mathrm{e}}$ arrondissement) en 1974.

11. - Elles sont peintes à la gouache et servent de documents préparatoires à des verrières fabriquées dans la technique traditionnelle du vitrail (verre à l'antique commandé pour l'essentiel à la verrerie de Saint-Just-Saint-Rambert dans la Loire, plus exceptionnellement, pour certains tons comme le rose à l'or, en Allemagne).

12. - Sur le premier exemple de mise en œuvre des «briques Huré », voir David, Véronique. Les vitraux de l'église Notre-Dame-des-Missions à Epinay-sur-Seine, In de Finance, Laurence. Un patrimoine de lumière (1830-2000). Paris, 2003. P. 287-293 (coll. Cahiers du Patrimoine, $\mathrm{n}^{\circ}$ 67).

13. - Doncoeur, Paul. L'art et le mobilier religieux moderne. In Etudes, 1929. P. 351. Ce vitrail avait également été exposé au Salon des Artistes Décorateurs (Gillet, E. Les vitraux de Marguerite Huré. Le Gaulois artistique. 28 mai 1929. P. 302).

14. - L'art chrétien. 1962, n²7-29. P. 69. Fonds d'atelier Huré/Lecamp.

15. - David, Véronique. De l'espoir retrouvé et des vitraux du Raincy. In de FINANCE, Laurence. Un patrimoine de lumière (1830-2000). Paris, 2003. P. 275-285. (coll. Cahiers du Patrimoine, $\left.n^{\circ} 67\right)$.

16. - Publié dans $\mathrm{ABC}$ artistique et littéraire. Octobre 1931, n 82. P. 262.

17. - Ses œuvres (créations et traductions de cartons ) sont dispersées dans 18 régions de France, surtout en Ile-de-France, en Picardie, en Basse-Normandie, en Bretagne et dans les Pays de la Loire.

18. - David, Véronique. Marguerite Huré. In L'Encyclopédie Perret, IFA. Paris, éditions Monum, 2002. P. 271-273.

19. - Déjà, avant son dépôt au Musée, ce fonds a pu alimenter les dossiers de protection Monuments historiques de la chapelle de l'ancien séminaire de Voreppe transformé en lycée professionnel, de l'église d'Aurillac dont Marguerite Huré a réalisé les vitraux en 1937 ainsi que des vitraux de l'ancienne Chapelle du Home d'enfants «Le clocher » à Villars-de-Lans mis en place en 1934 ( récemment déposés lors de la transformation du bâtiment en logements ). On peut citer également l'utilité de ce fonds pour les recherches d'Anne Tobé, présidente du CREHA, sur les vitraux du Plateau d'Assy, pour celles du Docteur Louise Campbell de l'Université de Warwick à Coventry, sur les ateliers d'artistes construits par Perret (voir : Perret and his ArtisClients : Architecture in the Age of Gold. Architectural history. 2002, vol. 45. P. 425-428) ou bien 
encore, celles de Fabienne Stahl-Escudero, dans le cadre de sa thèse sur Les décorations religieuses de Maurice Denis (1870-1943) entre les deux guerres, Université de Clermont-Ferrand (direction de J.P. Bouillon).

20. - Encyclopédie Perret, sous la direction de Jean-Louis Cohen, Joseph Abram et Guy Lambert, IFA, Paris, éditions du patrimoine Monum, Groupe Moniteur, Paris 2002.

21. - David, Véronique. Marguerite Huré et les peintres. Recherches en Histoire de l'art. 2002, $\mathrm{n}^{\circ}$ 1. ill. p. 159. Cordier, Valentine. A l'exposition coloniale. Glaces et Verres. Juin 1931, $\mathrm{n}^{\circ} 22 . \mathrm{P}$. 14.

22. - Le vitrail fut présenté sous le $n^{\circ} 24$ du catalogue.

23. - Voir en annexe l'inventaire du fonds "Marguerite Huré » établi par la conservation du Musée des années 30.

\section{AUTEURS}

\section{VÉRONIQUE DAVID}

Chercheur, cellule vitrail, Sous-direction des études, de la documentation et de l'Inventaire, Direction de l'architecture et du patrimoine. veronique.david@culture.gouv.fr

\section{CAROLE BOUVET (ÉPOUSE DE MERSSEMAN)}

Attachée de conservation. Chargée d'inventaire au musée des Années 30 de Boulogne-Billancourt caroledemersseman@gmail.com 